\title{
Automedicação em crianças: um problema de saúde pública
}

\author{
Children's self-medication: a public health concern \\ Grace Pfaffenbach ${ }^{1}$
}

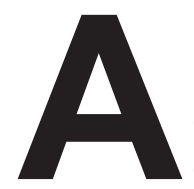

utilização de medicamentos tem sido descrita pela Organização Mundial da Saúde (OMS) como comercialização, distribuição, prescrição e uso de medicamentos em uma sociedade, com ênfase especial nas consequências médicas, sociais e econômicas ${ }^{(1)}$. Estudos a respeito do uso de medicamentos são relevantes em saúde pública, pois, desde o século 20 , quando os primeiros medicamentos começaram a ser produzidos, até os dias de hoje, seu processo se modificou e essas mudanças foram incorporadas nas legislações para registro e comercialização. O medicamento deixou de ser um produto de origem natural e artesanal para ser industrializado, passando a ser encarado com um bem de consumo $^{(2,3)}$. No entanto, o acesso universal aos serviços de saúde e aos medicamentos não vem acompanhando as necessidades em saúde de vários países. Paralelamente, as estruturas reguladoras de mercado em diversos países apresentam fragilidades e suas políticas de medicamentos mostram dificuldades de implementação(4).

O medicamento traz intrinsecamente um valor simbólico, que expressa o desejo de modificar o curso natural da doença. Dentro desse cenário, a automedicação se estabelece na tentativa de mitigar agravos em saúde, gerando irracionalidades no consumo, levando a consequências com impacto sanitário importantes para qualquer sistema de saúde. Atualmente, estima-se que cerca de $50 \%$ dos medicamentos vendidos, prescritos ou dispensados são inadequadamente consumidos ${ }^{(4,5)}$.

A automedicação pode, então, ser compreendida como o uso de medicamentos não-prescritos, muitas vezes de venda livre, com objetivo de "tratar" sintomas leves, sem consultar um médico(6), consistindo em um processo que ocorre por iniciativa do usuário ou de seu responsável para utilizar um produto com a crença de que este lhe trará benefícios no tratamento de doenças ou alívio de seus sintomas $^{(7-10)}$. Tal fenômeno tem implicação importante para crianças, nas quais o cuidado é exercido por seus responsáveis ${ }^{(11-14)}$.

Atualmente, o tema da automedicação em crianças tem sido abordado na literatura, mas carecem estudos que ampliem sua análise e permitam demonstrar intervenções efetivas. As motivações para a automedicação em crianças estão muitas vezes relacionadas à busca de alívio de sintomas como resfriado, febre e dor ${ }^{(14,15)}$. Estudos têm apontado que as principais classes terapêuticas utilizadas em crianças são anti-inflamatórios não-esteroidais, analgésicos e antibióticos ${ }^{(13,14,16-18)}$. A frequência da automedicação em crianças tem se mostrado elevada em vários estu$\operatorname{dos}^{(6,11-18)}$ e é fator preocupante quando parcela importante dessa população não recebe atenção adequada por parte dos serviços de saúde, ficando o cuidado restrito às decisões do cuidador.

O estudo publicado neste fascículo da Revista Paulista de Pediatria por Beckhauser et $a^{\left({ }^{(19)}\right.}$ aborda a questão da automedicação em crianças de famílias cobertas pela Estratégia Saúde da Família e traz rico material para reflexão sobre as práticas em saúde, bem como para o trabalho de educação com a comunidade.

\section{Referências bibliográficas}

1. World Health Organization. Technical report series: the selection of essential drugs. Geneve: WHO; 1977.

2. Sociedade Brasileira de Vigilância de Medicamentos. O que é uso racional de medicamentos. São Paulo: Sobravime; 2001.

3. Laporte JR, Tognoni G. Estudios de utilización de medicamentos y de farmacovigilancia. In: Laporte JR, Tognoni G, editors. Principios de epidemiología del medicamento. 2nd ed. Barcelona: Ediciones Científicas y Técnicas; 1993. p. 1-24.

4. World Health Organization. The world medicines situation. Geneve: WHO; 2004.

5. World Health Organization. Conference of experts on the rational use of drugs: the rational use of drugs. Geneva: WHO; 1987.

6. Du Y, Knopf H. Self-medication among children and adolescents in Germany: results of the National Health Survey for Children and Adolescents (KiGGS). Br J Clin Pharmacol 2009;68:599-608.

7. Hughes CM, McElnay JC, Fleming GF. Benefits and risks of self medication Drug Saf 2001;24:1027-37.

8. World Health Organization. Guidelines for the medical assessment of drugs for use in self-medication. Copenhagen: WHO; 1986.

9. World Health Organization. The benefits and risks of self-medication. WHO Drug Information 2000;14:1-2.

10. Arrais PS, Coelho HL, Batista MC, Carvalho ML, Righi RE, Arnau JM. Perfil da automedicação no Brasil. Rev Saude Publica 1997;31:71-7.

11. Bricks LF, Leone C. Utilização de medicamentos por crianças atendidas em creches. Rev Saude Publica 1996;30:527-35.

12. Mendes Z, Martins AP, Miranda AC, Soares MA, Ferreira AP, Nogueira A. Self-medication prevalence in a Portuguese Urban Area. RBCF 2004;40:21-5.

13. da Silva $\mathrm{CH}$, Giugliani ER. Consumption of medicines among adolescent students: a concern. J Pediatr (Rio J) 2004;80:326-32. 
14. Pereira FS, Bucaretchi F, Stephan C, Cordeiro R. Self-medication in children and adolescents. J Pediatr (Rio J) 2007;83:453-8.

15. Bi P, Tong S, Parton KA. Family self-medication and antibiotics abuse for children and juveniles in a Chinese city. Soc Sci Med 2000;50:1445-50.

16. Béria JU, Victora CG, Barros FC, Teixeira AB, Lombardi C. [Epidemiology of drug consumption in children of a urban center of the southern region of Brazil]. Rev Saude Publica 1993;27:95-104.

17. Morales-Carpi C, Julve Chover N, Carpi Lobatón R, Estañ L, Rubio E, Lurbe
$E$ et al. Drugs used in paediatric outpatients: do we have enough information available? An Pediatr (Barc) 2008;68:439-46.

18. de Carvalho DC, Trevisol FS, Menegali BT, Trevisol DJ. Drug utilization among children aged zero to six enrolled in day care centers of Tubarão, Santa Catarina, Brazil. Rev Paul Pediatr 2008;26:238-44.

19. Beckhauser GC, Souza JM, Valgas C, Piovezan AP, Galato D. Utilização de medicamentos na Pediatria: uma investigação sobre a prática de automedicação em crianças por seus responsáveis. Rev Paul Pediatr 2010;28:262-8.
Instituição: Hospital de Clínicas da Faculdade de Ciências Médicas da Universidade Estadual de Campinas (Unicamp), Campinas, SP, Brasil

'Doutora; Membro do Núcleo de Avaliação de Tecnologias em Saúde e da Comissão de Farmácia e Terapêutica do Hospital de Clínicas da Faculdade de Ciências Médicas da Unicamp, Campinas, SP, Brasil
Endereço para correspondência:

Grace Pfaffenbach

Rua Vital Brasil, 251 - Cidade Universitária Zeferino Vaz

CEP: 13083-888 - Campinas, SP

E-mail: grace@hc.unicamp.br

Conflito de interesse: nada a declarar

Recebido em: 8/6/2010 